\title{
The Study of Reservation Scheme for Managing Parking Cruising
}

\author{
Duo $\mathrm{XU}^{\mathrm{a}}$ and Tian-Chao GUAN \\ School of Traffic and Transportation, Beijing Jiaotong University, Beijing, China
}

\begin{abstract}
Cruising for parking is a main contributor to traffic jam while the related researches are few. Assume travelers are well-informed, this paper first analyses user equilibrium (UE) in no parking fee scheme with different scarcity of parking spaces. We get a conclusion that information fails to solve traffic problems effectively in absent of control. Followed by the principle of cape and trade, the paper proposes parking reservation scheme which combining guidance (information) with tolling (control). Example shows that system cost reduces significantly after the implementation of the parking reservation scheme.
\end{abstract}

\section{Introduction}

Parking is a growing problem in many large cities around world. Although the average parking time of a car is 23 hours a day [1], looking for vacant parking spaces accounts for about $30 \%-50 \%$ of the rest 1 hour and nearly $70 \%$ of the travel cost [2]. Study found that most drivers will spend 3.5 to 14 minutes before they get parked, and cruising for parking is a major contributor (30\% on average) to traffic jam in downtown [3].

Demand management is an effective way to solve this problem. Vickrey first proposed that parking resources should be priced in accordance with the social marginal cost [4]. With the development of intelligent transportation systems, researches are focusing on time-varying toll, parking guidance and information, tradable credit, etc. [5]-[7]. However, the issue of searching has been less concerned about, and only information could not solve that problem. So we propose parking reservation scheme combining guidance (information) with tolling (control) to reduce system cost.

\section{Basic settings}

Consider a traffic corridor connecting residential area and central business district(CBD). Morning commuters drive from home to workplace everyday. The corridor contains an expressway with a bottleneck which has a finite capacity. Queue will emerge when traffic flow rate exceeds bottleneck capacity. Parking lots are near CBD. Cars can choose any free spot to park. After drives towards downtown, each commuter looks for an vacant parking spot, then walks to the workplace. All of the commuters are assumed to have a common work beginning time $t^{*}$, and both early and late arrival will be penalized.

\footnotetext{
${ }^{a}$ Corresponding author: ringloveyan1987@yahoo.com
} 
The general cost of each traveler contains travel cost, searching cost and schedule delay cost. All commuters experience the same time on driving over the expressway and walking to the workplace. For simplicity, we ignore the free flow commute time and walk time [8].

Denote $T(t)$ be the travel time (the queuing time only). $T(t)=q(t) / s$, where $q(t)$ is the queue length at the bottleneck at time $t$, and $s$ is the capacity of the bottleneck. The change of the queue can be express as

$$
\frac{\mathrm{d} q(t)}{\mathrm{d} t}=\left\{\begin{array}{l}
r(t)-s, r(t)>s \text { or } q(t)>0 \\
0, r(t) \leq s \text { and } q(t)=0
\end{array}\right.
$$

Where $r(t)$ is the arriving rate of the traffic flow at bottleneck at time $t$ (also can be seen as departure time). The queue will develop when traffic flow rate exceeds bottleneck capacity. Let $\alpha$ be the value of time of commuters. The travel cost of the commuter who departs at time $t$ is

$$
C_{\mathrm{q}}(t)=\alpha T(t)
$$

Set the total number of parking spots is $k$ and the total number of commuters is $N . n(t)$ is the occupied parking spots at time $t$, which satisfies $n(t) \leq N \leq k$. The probability that a randomly sampled spot is free is given by $[k-n(t)] / k[8]$. Assume that a driver who checks whether or not a spot is free incurs a time cost $\lambda$, the expect time he will spend to get a free spot is $\lambda k /[k-n(t)]$. Therefore the expect searching cost to an individual is

$$
C_{\mathrm{s}}(t)=\frac{\alpha \lambda k}{k-n(t)}
$$

Let the $\beta$ and $\gamma$ be the marginal cost of early arrival and late arrival respectively, where $\gamma>\alpha>\beta$. The schedule delay cost of the driver who departs at time $t$ is given by

$$
C_{\mathrm{d}}(t)=\beta \max \left\{0, t^{*}-t-T(t)\right\}+\gamma \max \left\{0, T(t)+t-t^{*}\right\}
$$

Denote $p(t)$ be the parking fee of the parking lot near CBD at time $t$. The generalized travel cost of the travelers who depart at $t$ is given by

$$
C(t)=C_{\mathrm{q}}(t)+C_{\mathrm{s}}(t+T(t))+C_{\mathrm{d}}(t)+p(t+T(t))
$$

\section{User equilibrium without parking fee}

We suppose travelers are aware of parking information at any time via either online information system or sufficient experience. We first study on travelers' parking behaviors and corresponding parking flow pattern under no parking fee scheme. We define user equilibrium as, 1. All travelers' generalized travel costs are same. 2. No traveler can unilaterally change his parking choice and/or departure time choices to reduce his generalized travel cost.

\subsection{Equilibrium analysis}

Travelers are trying to minimize their cost. Let $t_{\mathrm{e}}$ and $t_{\mathrm{s}}$ be the departure time of the first and the last traveler. The first and the last traveler will not encounter queuing [9]. We assume that each of the searching cost and the queuing cost accounts for a certain part of the equilibrium cost. Therefore, the bottleneck will be at fully service capacity during the entire departure period which can be given as

$$
t_{\mathrm{e}^{-}} t_{\mathrm{s}}=N / s
$$

The cost of the first and the last traveler, as well as the traveler without schedule delay are 


$$
\begin{gathered}
C\left(t_{\mathrm{s}}\right)=\beta\left(t^{*}-t_{\mathrm{s}}-\lambda\right)+\alpha \lambda \\
C\left(t_{\mathrm{e}}\right)=\gamma\left(t_{\mathrm{e}}+\frac{\lambda k}{k-N+1}-t^{*}\right)+\frac{\alpha \lambda k}{k-N+1} \\
C\left(t_{\mathrm{n}}\right)=\alpha\left(t^{*}-t_{\mathrm{n}}\right)
\end{gathered}
$$

Where $t_{\mathrm{n}}$ is the departing time of the traveler without schedule delay. Denote $\pi$ is the equilibrium cost.

$$
\begin{gathered}
t_{\mathrm{s}}=t^{*}+\frac{\alpha-\beta}{\beta} \lambda-\frac{\pi}{\beta} \\
t_{\mathrm{e}}=t^{*}+\frac{\pi}{\gamma}-\frac{\alpha+\gamma}{\gamma} \frac{\lambda k}{k-N+1} \\
t_{\mathrm{n}}=t^{*}-\frac{\pi}{\alpha}
\end{gathered}
$$

Combine (10) and (11) with (6), the equilibrium cost is

$$
\pi=\frac{\beta \gamma}{\beta+\gamma} \frac{N}{s}+\frac{\beta(\alpha+\gamma)}{\beta+\gamma} \frac{\lambda k}{k-N+1}+\frac{\gamma(\alpha-\beta)}{\beta+\gamma} \lambda
$$

Accroding to (5), the queuing time is

$$
T(t)= \begin{cases}\frac{\pi-\beta\left(t^{*}-t\right)}{\alpha-\beta}-\frac{\lambda k}{k-\left(t-t_{\mathrm{s}}\right) s+1} & t_{\mathrm{s}} \leq t \leq t_{\mathrm{n}} \\ \frac{\pi-\gamma\left(t-t^{*}\right)}{\alpha-\beta}-\frac{\lambda k}{k-\left(t-t_{\mathrm{s}}\right) s+1} & t_{\mathrm{n}} \leq t \leq t_{\mathrm{e}}\end{cases}
$$

Combine (14) with (1), the departing rate of the travelers is given by

$$
r(t)= \begin{cases}\frac{\alpha s}{\alpha-\beta}-\frac{\lambda k s^{2}}{\left[k-\left(t-t_{\mathrm{s}}\right) s+1\right]^{2}} & t_{\mathrm{s}} \leq t \leq t_{\mathrm{n}} \\ \frac{\alpha s}{\alpha+\gamma}-\frac{\lambda k s^{2}}{\left[k-\left(t-t_{\mathrm{s}}\right) s+1\right]^{2}} & t_{\mathrm{n}} \leq t \leq t_{\mathrm{e}}\end{cases}
$$

\subsection{Case study}

In this subsection, we provide an example to illustrate commuters' travelling behavior and parking distribution under the influences of queuing cost and searching cost. Assuming the work beginning time is 9:00 and the total number of commuters $N$ is 1000 . Bottleneck capacity is 2000 per hour. The value of unit travel time $\alpha$ and the marginal schedule delay cost $\beta$ and $\gamma$ is 40 per hour, 20 per hour and 80 per hour, respectively. Searching time $\lambda$ is set as 0.005 hour [10]. To reveal the characteristics of the system under different searching costs, we set the total number of parking spots $k$ as a variable. 
Figure. 1 depicts the searching cost of the last traveler $\left(C_{\mathrm{s}}\left(t_{\mathrm{e}}\right)=\alpha \lambda k /(k-N+1)\right)$ with different occupancy rate $(N / k)$. When the parking spaces are adequate (parking occupancy rate is lower than 0.8 ), searching cost is low, and its growth trend is slow. In contrast, the searching cost grows rapidly.

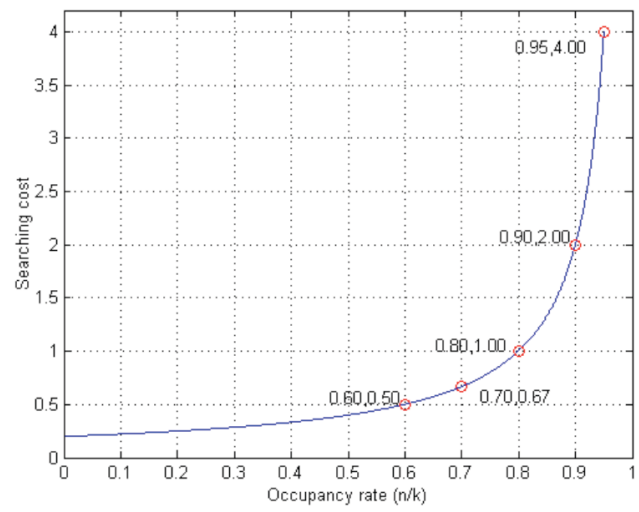

Figure 1. The relationship between occupancy rate and searching cost

In this example, the maximum parking occupancy rate that satisfies late arrivals is 0.94 . Accroding to (15), the departure rate of early arrivals is greater than bottleneck capacity, the queue is accumlating and the travel time is on the rise. On the opposite, the departure rate of late arrivals is less than bottleneck capacity, the queue is dissipating and the travel time is declining.

Figure 2 shows the cumulative number of departure and arrival in the condition of parking spaces are adequate $(N / k \rightarrow 0$, black dotted line) and inadequate $(N / k=0.94$, blue solid line $)$, respectively. The ordinate represents the number of trips while the abscissa represents the time ( 30 to 65 represents 8: 30 to 9: 05). Points $A 2$ and $B 2$ are the traveler who arrives on time in both cases. Curve $A 1 A 2$ and line $B 1 B 2$ characterize the departure rate of the early traveler while curve $A 2 A 3$ and line $B 2 B 3$ depict the departure rate of the late traveler. Line $A 1 A 3$ and $B 1 B 3$ are the bottleneck capacity. The difference between curves $A 1 A 2 A 3$ and $B 1 B 2 B 3$ in vertical is the queue length at the bottleneck and the difference in the horizontal is queuing time.
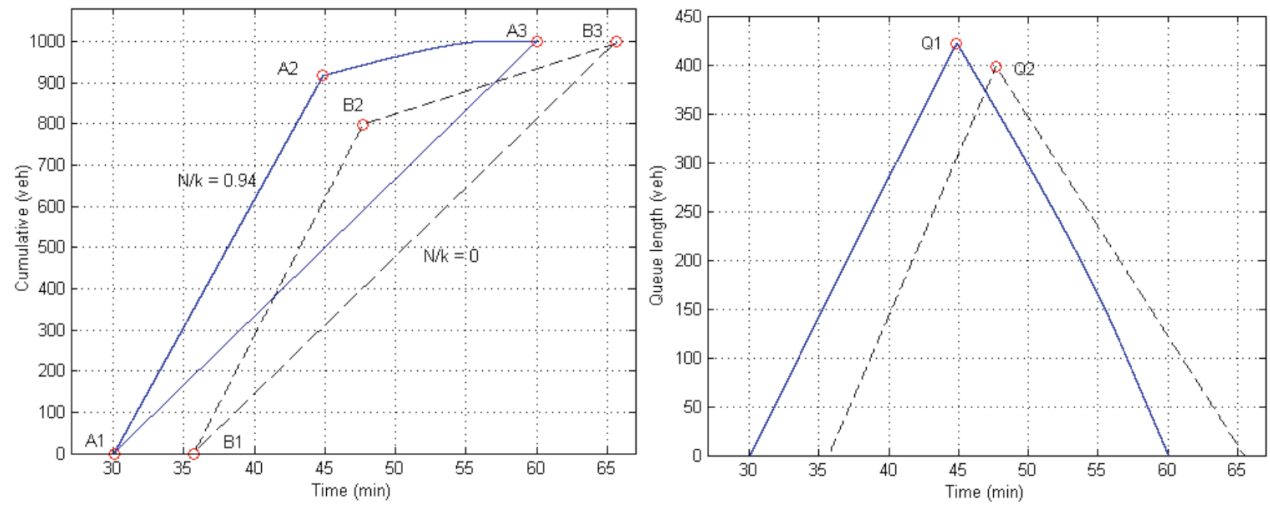

Figure 2. The departure (arrival) cumulative number and the queue length (queuing time) of in two cases

As can be easily seen, with the increase of the scarcity of parking spaces, 1 . All the travelers depart earlier. The number of early arrivals increases while the number of late arrivals decreases. 2 . The departure rate is on decreasing trend as the number of occupied parking spots increasing, and declines 
to zero when the parking lot approaches the full amount. 3. The maximum queuing length and the maximum queuing time at the bottleneck raises because of the more concentrated departing.

Figure 3 shows the departure time of the first (black solid line) and the last (blue solid line) traveler as well as the traveler with no schedule delay (red solid line) when $N / k$ changes from 0 to 0.94. As we can see, 1 . Three departure times change in a small range when $N / k$ is lower than 0.8 and move ahead obviously when $N / k$ is close to 0.94 . It indicates the more scarce parking spaces are,the greater it impacts to travelers' departure time. 2 . The departure time of the traveler with no schedule delay moves slowly compare with the first and the last traveler both. It reflects the departing order of that traveler is increasing when $N / k$ grows.

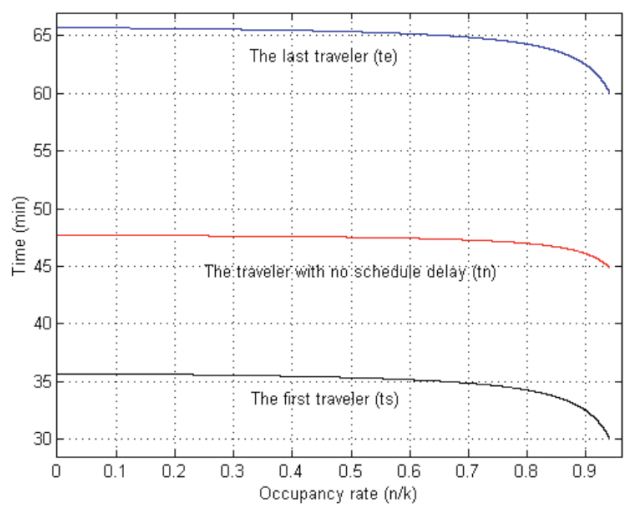

Figure 3. Departure time of the first (on time/ last) traveler in different $N / k$

\section{System optimum in parking reservation scheme}

Through subsection 3.4, we get the conclusion that travelers' behavior is affected by the scarcity of the parking spaces. The deadweight loss which contains queuing cost and searching cost grows obviously when commuters number is near to the number of spots in parking lot.

Tolling is an effective way to control the parking demand and distribution as well as minimizes the deadweight loss.Early researchs used to save the system cost by converting a portion of deadweight loss to the income of the parking lot through tolling, but they do not reduce the travelers' cost. Accroding to (3), searching cost becomes the main contributor as the parking lot is scarce which is consistent with the reality. Therefore, to further eliminate the searching cost so as to reduce the equilibrium cost is the goal of the traffic manager.

Parking reservation scheme, which establish a one-to-one link between parkers and parking spots before a trip, can effectively eliminates searching by avoiding the mutual influence among parkers. Reservations can also be designed according to the bottleneck capacity constraint so as to eliminate queuing [8].

The object of the system is on the premise of minimize travelers' equilibrium cost, parking managers should increase parking fees as much as possible.

$$
\begin{array}{ll}
\text { (U) } & \min . C(t) \\
& \text { s.t. } t \geq 0 \\
\text { (L) } & \max . p(t+T(t)) \\
& \text { s.t. } t \geq 0
\end{array}
$$

The equilibrium cost and the optimal parking fees are 


$$
\begin{aligned}
& \pi=\frac{\beta \gamma}{\beta+\gamma} \frac{N}{S}+\alpha \lambda \\
& p(t)=\left\{\begin{array}{cl}
\frac{\beta \gamma}{\beta+\gamma} \frac{N}{s}+\alpha \lambda-\beta\left(t^{*}-t\right) & t_{\mathrm{s}} \leq t \leq t^{*} \\
\frac{\beta \gamma}{\beta+\gamma} \frac{N}{s}+\alpha \lambda-\gamma\left(t-t^{*}\right) & t^{*} \leq t \leq t_{\mathrm{e}}
\end{array}\right.
\end{aligned}
$$

Follow the example from the previous section. Let $N / k$ equals to 0.94 . We can see travelers' behavior before and after the implementation of parking reservation scheme from Figure 4 .

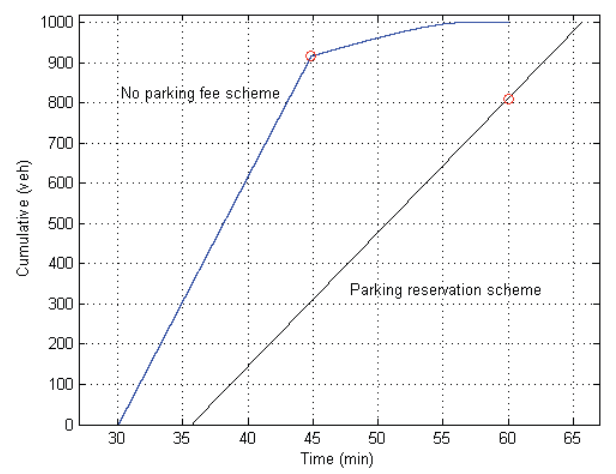

Figure 4. The cumulative number of departure and arrival in schemes

Compare with the no parking fees scheme, all travelers in parking reservation scheme can depart later on different extend. The equilibrium travel cost is decreased from 10.02 to 8.20 , reduced by $18.16 \%$. Parking fees is 4.10 per capita, and accounts for a half of the total cost. Because of the income of the parking fees, the system cost is 5.10 , reduced by $59.08 \%$.

\section{Conclusion}

In this paper, we analyze the process of how morning commuters choose their departure time under UE condition as well as how the searching cost influences their behavior and contributes to larger deadweight loss. Through an example, we found the system performance in parking reservation scheme is prior to in no parking fee scheme due to the significant reduction of both the cost of user and the deadweight loss of system.

\section{References}

1. Z. Qian, F. E. Zhang, Transportation Research Part A, 45 (9), 861-879 (2011)

2. R. Arnott, J. Rowse, Journal of Urban Economics, 45, 97-124 (1999)

3. D. Shoup, The high cost of free parking (American Planning Association, 2005)

4. W. S. Vickrey, American Economic Review, 59 (2), 251-261 (1969)

5. H. Yang, X. Wang, Transportation Research Part B, 45 (3), 580-594 (2011)

6. Z. Qian, R. Rajagopal, Procedia - Social and Behavioral Sciences, 80, 779-805 (2013)

7. G. S. Muller, M. Xu, Transport Reviews, 34 (2) , 128-149 (2014)

8. W. Liu, H. Yang, Y. F. Yin, Transportation Research Part C, 44, 185-201 (2014) 
9. Z. X. Wu, H. J. Huang, System Engineering Theory and Practice, 1, 130-135(2000)

10. S. Anderson, A. de Palma, Urban Economics, 55, 1-20 (2004) 